\title{
The warp drive: hyper-fast travel within general relativity.
}

\author{
Miguel Alcubierre* \\ Department of Physics and Astronomy, University of Wales, \\ College of Cardiff, P.O. Box 913, Cardiff CF1 3YB, UK.
}

PACS numbers : 0420, 0490.

\begin{abstract}
It is shown how, within the framework of general relativity and without the introduction of wormholes, it is possible to modify a spacetime in a way that allows a spaceship to travel with an arbitrarily large speed. By a purely local expansion of spacetime behind the spaceship and an opposite contraction in front of it, motion faster than the speed of light as seen by observers outside the disturbed region is possible. The resulting distortion is reminiscent of the "warp drive" of science fiction. However, just as it happens with wormholes, exotic matter will be needed in order to generate a distortion of spacetime like the one discussed here.
\end{abstract}

Published in: $\quad$ Class. Quantum Grav. 11-5, L73-L77 (1994).

*Present address: Max Planck Institut für Gravitationsphysik, Albert Einstein Institut, Schlaatzweg 1, D-14473 Potsdam, Germany. 
When we study special relativity we learn that nothing can travel faster than the speed of light. This fact is still true in general relativity, though in this case one must be somewhat more precise: in general relativity, nothing can travel locally faster than the speed of light.

Since our everyday experience is based on an Euclidean space, it is natural to believe that if nothing can travel locally faster than light then given two places that are separated by a spatial proper distance $D$, it is impossible to make a round trip between them in a time less than $2 D / c$ (where $c$ is the speed of light), as measured by an observer that remains always at the place of departure. Of course, from our knowledge of special relativity we know that the time measured by the person making the round trip can be made arbitrarily small if his (or her) speed approaches that of light. However, the fact that within the framework of general relativity and without the need to introduce nontrivial topologies (wormholes), one can actually make such a round trip in an arbitrarily short time as measured by an observer that remained at rest will probably come as a surprise to many people.

Here I wish to discuss a simple example that shows how this can be done. The basic idea can be more easily understood if we think for a moment in the inflationary phase of the early Universe, and consider the relative speed of separation of two comoving observers. It is easy to convince oneself that, if we define this relative speed as the rate of change of proper spatial distance over proper time, we will obtain a value that is much larger than the speed of light. This doesn't mean that our observers will be travelling faster than light: they always move inside their local light-cones. The enormous speed of separation comes from the expansion of spacetime itself.円

\footnotetext{
${ }^{1}$ This superluminal speed is very often a source of confusion. It is also a very good example of how an intuition based on special relativity can be deceiving when one deals with dynamical spacetimes.
} 
The previous example shows how one can use an expansion of spacetime to move away from some object at an arbitrarily large speed. In the same way, one can use a contraction of spacetime to approach an object at any speed. This is the basis of the model for hyperfast space travel that I wish to present here: create a local distortion of spacetime that will produce an expansion behind the spaceship, and an opposite contraction ahead of it. In this way, the spaceship will be pushed away from the Earth and pulled towards a distant star by spacetime itself. One can then invert the process to come back to Earth, taking an arbitrarily small time to complete the round trip.

I will now introduce a simple metric that has precisely the characteristics mentioned above. I will do this using the language of the $3+1$ formalism of general relativity [1, 2], because it will permit a clear interpretation of the results. In this formalism, spacetime is described by a foliation of spacelike hypersurfaces of constant coordinate time $t$. The geometry of spacetime is then given in terms of the following quantities: the 3 -metric $\gamma_{i j}$ of the hypersurfaces, the lapse function $\alpha$ that gives the interval of proper time between nearby hypersurfaces as measured by the "Eulerian" observers (those whose four-velocity is normal to the hypersurfaces), and the shift vector $\beta^{i}$ that relates the spatial coordinate systems on different hypersurfaces. Using these quantities, the metric of spacetime can be written as: 9

$$
\begin{aligned}
d s^{2} & =-d \tau^{2}=g_{\alpha \beta} d x^{\alpha} d x^{\beta} \\
& =-\left(\alpha^{2}-\beta_{i} \beta^{i}\right) d t^{2}+2 \beta_{i} d x^{i} d t+\gamma_{i j} d x^{i} d x^{j}
\end{aligned}
$$

Notice that as long as the metric $\gamma_{i j}$ is positive definite for all values of $t$ (as it should in order for it to be a spatial metric), the spacetime is guaranteed to be globally

\footnotetext{
${ }^{2}$ In the following greek indices will take the values $(0,1,2,3)$ and latin indices the values $(1,2,3)$.
} 
hyperbolic. Any spacetime that can be described in the language of the $3+1$ formalism will therefore have no closed causal curves.

Let us now assume that our spaceship moves along the $x$ axis of a cartesian coordinate system. We want to find a metric that will "push" the spaceship along a trajectory described by an arbitrary function of time $x_{s}(t)$. A metric that has this property is given by $(G=c=1)$ :

$$
\begin{aligned}
\alpha & =1, \\
\beta^{x} & =-v_{s}(t) f\left(r_{s}(t)\right), \\
\beta^{y} & =\beta^{z}=0, \\
\gamma_{i j} & =\delta_{i j},
\end{aligned}
$$

where:

$$
v_{s}(t)=\frac{d x_{s}(t)}{d t}, \quad r_{s}(t)=\left[\left(x-x_{s}(t)\right)^{2}+y^{2}+z^{2}\right]^{1 / 2}
$$

and where $f$ is the function:

$$
f\left(r_{s}\right)=\frac{\tanh \left(\sigma\left(r_{s}+R\right)\right)-\tanh \left(\sigma\left(r_{s}-R\right)\right)}{2 \tanh (\sigma R)}
$$

with $R>0$ and $\sigma>0$ arbitrary parameters. Notice that for large $\sigma$ the function $f(r)$ approaches very rapidly a "top hat" function:

$$
\lim _{\sigma \rightarrow \infty} f\left(r_{s}\right)= \begin{cases}1 & \text { for } r_{s} \in[-R, R] \\ 0 & \text { otherwise }\end{cases}
$$

With the above definitions, the metric (11) can be rewritten as:

$$
d s^{2}=-d t^{2}+\left(d x-v_{s} f\left(r_{s}\right) d t\right)^{2}+d y^{2}+d z^{2}
$$


It is easy to understand the geometry of our spacetime from the previous expressions. First, from equation (5) we see that the 3-geometry of the hypersurfaces is always flat. Moreover, the fact that the lapse is given by $\alpha=1$ implies that the timelike curves normal to these hypersurfaces are geodesics, i.e., the Eulerian observers are in free fall. Spacetime, however, is not flat due to the presence of a non-uniform shift. Nevertheless, since the shift vector vanishes for $r_{s} \gg R$, we see that at any time $t$ spacetime will be essentially flat everywhere except within a region with a radius of order $R$, centered at the point $\left(x_{s}(t), 0,0\right)$.

Since the 3-geometry of the hypersurfaces is flat, the information about the curvature of spacetime will be contained in the extrinsic curvature tensor $K_{i j}$. This tensor describes how the three-dimensional hypersurfaces are embedded in four-dimensional spacetime, and is defined as:

$$
K_{i j}=\frac{1}{2 \alpha}\left(D_{i} \beta_{j}+D_{j} \beta_{i}-\frac{\partial g_{i j}}{\partial t}\right)
$$

where $D_{i}$ denotes covariant differentiation with respect to the 3 -metric $\gamma_{i j}$. From the form of $\alpha$ and $\gamma_{i j}$, is not difficult to see that this expression reduces to:

$$
K_{i j}=\frac{1}{2}\left(\partial_{i} \beta_{j}+\partial_{j} \beta_{i}\right)
$$

The expansion $\theta$ of the volume elements associated with the Eulerian observers is given in terms of $K_{i j}$ as:

$$
\theta=-\alpha \operatorname{Tr} K
$$

From this expression it is not difficult to show that:

$$
\theta=v_{s} \frac{x_{s}}{r_{s}} \frac{d f}{d r_{s}}
$$

Figure1 shows a graph of $\theta$ as a function of $x$ and $\rho=\left(y^{2}+z^{2}\right)^{1 / 2}$, in the particular case when $\sigma=8$ and $R=v_{s}=1$. The center of the perturbation 
corresponds to the spaceship's position $x_{s}(t)$. We clearly see how the volume elements are expanding behind the spaceship, and contracting in front of it.

To prove that the trajectory of the spaceship is indeed a timelike curve, regardless of the value of $v_{s}(t)$, we substitute $x=x_{s}(t)$ in the metric (8). It is then easy to see that for the spaceship's trajectory we will have:

$$
d \tau=d t
$$

This implies not only that the spaceship moves on a timelike curve, but also that its proper time is equal to coordinate time. Since coordinate time is also equal to the proper time of distant observers in the flat region, we conclude that the spaceship suffers no time dilation as it moves. It is also straightforward to prove that the spaceship moves on a geodesic. This means that even though the coordinate acceleration can be an arbitrary function of time, the proper acceleration along the spaceship's path will always be zero. Moreover, it is not difficult to convince oneself that when the parameter $\sigma$ is large, the tidal forces in the immediate vicinity of the spaceship are very small (provided that $R$ is larger than the size of the spaceship). Of course, in the region where $r_{s} \simeq R$ the tidal forces can be very large indeed.

To see how one can use this metric to make a round trip to a distant star in an arbitrary small time, let us consider the following situation: Two stars $A$ and $B$ are separated by a distance $D$ in flat spacetime. At time $t_{0}$, a spaceship starts to move away from $A$ at a speed $v<1$ using its rocket engines. The spaceship then stops at a distance $d$ away from $A$. I will assume that $d$ is such that:

$$
R \ll d \ll D
$$

It is at this point that a disturbance of spacetime of the type described, centered 
at the spaceship's position, first appears. This disturbance is such that the spaceship is pushed away from $A$ with a coordinate acceleration that changes rapidly from 0 to a constant value $a$. Since the spaceship is initially at rest $\left(v_{s}=0\right)$, the disturbance will develop smoothly from flat spacetime (see equation (8)).

When the spaceship is halfway between $A$ and $B$, the disturbance is modified in such a way that the coordinate acceleration changes rapidly from $a$ to $-a$. If the coordinate acceleration in the second part of the trip is arranged in such a way as to be the opposite to the one we had in the first part, then the spaceship will eventually find itself at rest at a distance $d$ away from $B$, at which time the disturbance of spacetime will disappear (since again $\left.v_{s}=0\right)$. The journey is now completed by moving again through flat spacetime at a speed $v$.

If each of the changes in acceleration are very rapid, the total coordinate time $T$ elapsed in the one way trip will be essentially given by:

$$
T=2\left[\frac{d}{v}+\sqrt{\frac{D-2 d}{a}}\right] .
$$

Since both stars remain in flat space, their proper time is equal to coordinate time. The proper time measured on the spaceship, on the other hand, will be:

$$
\tau=2\left[\frac{d}{\gamma v}+\sqrt{\frac{D-2 d}{a}}\right],
$$

with $\gamma=\left(1-v^{2}\right)^{-1 / 2}$. We see then that the time dilation comes only from the initial and final stages of the trip, when the spaceship moves through flat spacetime. Now, if

\footnotetext{
${ }^{3}$ The two constant-velocity legs at the beginning and end of the journey are not crucial for the argument that I wish to present here. I only introduce them in order to guarantee that the two stars will remain unaffected by the disturbance of spacetime $(R \ll d)$, and can therefore be used as unperturbed "clocks" with which to compare the proper time on board the spaceship.
} 
condition (14) holds, we will have:

$$
\tau \simeq T \simeq 2 \sqrt{\frac{D}{a}}
$$

It is now clear that $T$ can be made as small as we want by increasing the value of $a$. Since a round trip will only take twice as long, we find that we can be back in star $A$ after an arbitrarily small proper time, both from the point of view of the spaceship and from the point of view of the star. The spaceship will then be able to travel much faster than the speed of light. However, as we have seen, it will always remain on a timelike trajectory, that is, inside its local light-cone: light itself is also being pushed by the distortion of spacetime. A propulsion mechanism based on such a local distortion of spacetime just begs to be given the familiar name of the "warp drive" of science fiction.

The metric I have just described has one important drawback, however: it violates all three energy conditions (weak, dominant and strong [3]). Both the weak and the dominant energy conditions require the energy density to be positive for all observers. If one calculates the Einstein tensor from the metric (\$), and uses the fact that the four-velocity of the Eulerian observers is given by:

$$
n^{\alpha}=\frac{1}{\alpha}\left(1,-\beta^{i}\right), \quad n_{\alpha}=-(\alpha, 0)
$$

then one can show that these observers will see an energy density given by:

$$
T^{\alpha \beta} n_{\alpha} n_{\beta}=\alpha^{2} T^{00}=\frac{1}{8 \pi} G^{00}=-\frac{1}{8 \pi} \frac{v_{s}^{2} \rho^{2}}{4 r_{c}^{2}}\left(\frac{d f}{d r_{s}}\right)^{2}
$$

The fact that this expression is everywhere negative implies that the weak and dominant energy conditions are violated. In a similar way one can show that the strong energy condition is also violated. 
We see then that, just as it happens with wormholes, one needs exotic matter to travel faster than the speed of light. However, even if one believes that exotic matter is forbidden classically, it is well known that quantum field theory permits the existence of regions with negative energy densities in some special circumstances (as, for example, in the Casimir effect [4). The need of exotic matter therefore doesn't necessarily eliminate the possibility of using a spacetime distortion like the one described above for hyper-fast interstellar travel.

As a final comment, I will just mention the fact that even though the spacetime described by the metric (8) is globally hyperbolic, and hence contains no closed causal curves, it is probably not very difficult to construct a spacetime that does contain such curves using a similar idea to the one presented here.

The author wishes to thank Bernard F. Schutz and Gareth S. Jones for many useful comments.

\section{References}

[1] C.W. Misner, K.S. Thorne and J.A. Wheeler, Gravitation, W.H. Freeman, 1973.

[2] J.W. York, "Kinematics and Dynamics of General Relativity" in: Sources of Gravitational Radiation, ed. L.L. Smarr, pp. 83-126, Cambridge University Press, 1979.

[3] S.W. Hawking and G.F.R. Ellis, The Large Scale Structure of Spacetime, Cambridge University Press, 1973.

[4] B.S. DeWitt, in General Relativity: An Einstein Centenary Survey, edited by S.W. Hawking and W. Israel, Cambridge University Press, Cambridge, 1979. 


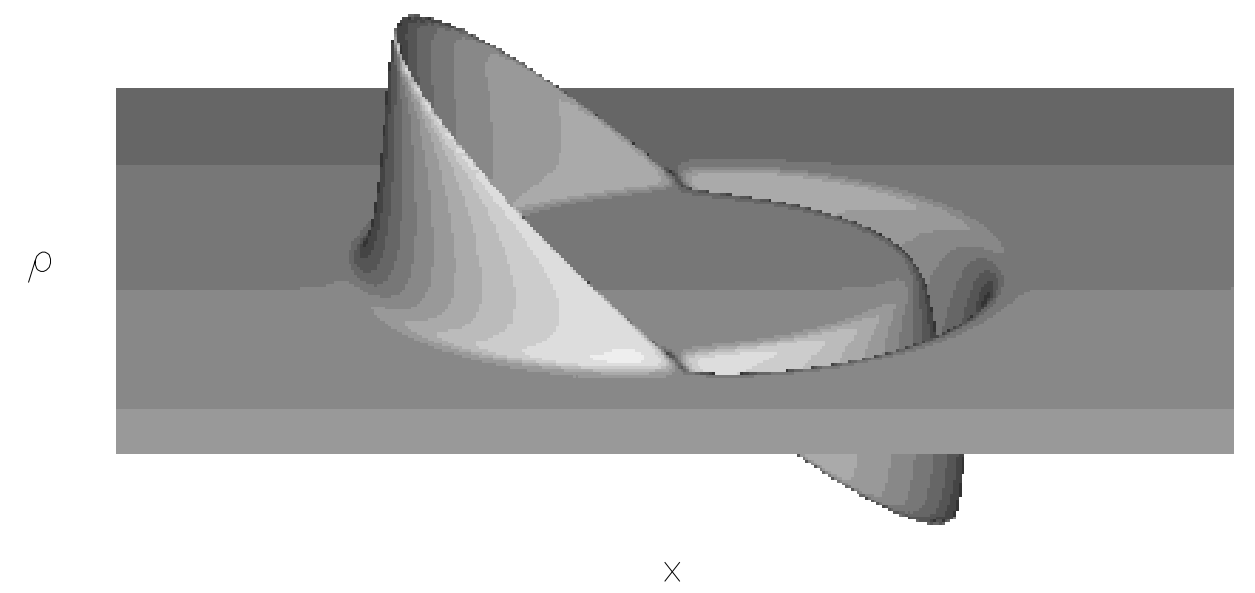

Figure 1: Expansion of the normal volume elements. 